\title{
Complicaciones relacionadas con el cateterismo cardíaco pediátrico y cardiopatías congénitas
}

\author{
Complications associated to pediatric cardiac catheterization and congenital heart \\ diseases
}

\author{
Rafael Lince-Varela1*, Diana Restrepo², Manuela Lince ${ }^{3}$, David Muñoz ${ }^{3}$, Federico Vásquez ${ }^{3}$, \\ José M. Quijano3, Laura Hincapié3, Juan F. Hinestroza3 ${ }^{3}$, Mariana Velásquez ${ }^{3}$ y Juliana Bedoya ${ }^{3}$ \\ ${ }^{1}$ Servicio de Cardiología Pediátrica, Clínica CardioVID, Medellín; ${ }^{2}$ Facultad de Medicina, Universidad CES, Medellín; ${ }^{3}$ Facultad de Medicina, \\ Universidad CES, Medellín. Antioquia, Colombia
}

\begin{abstract}
Resumen
Introducción: Las cardiopatías congénitas son las anomalías congénitas más comunes en recién nacidos con una prevalencia de $1 \%$. El cateterismo cardíaco diagnóstico y terapéutico ha revolucionado el tratamiento de estas enfermedades, aunque puede acompañarse de complicaciones. Objetivo: Describir la incidencia y el tipo de complicaciones vinculadas con el cateterismo cardíaco pediátrico en un centro de referencia para cardiopatías congénitas. Método: Estudio observacional retrospectivo, con intención analítica a partir de registros de cateterismos cardíacos realizados a pacientes con cardiopatías congénitas y adquiridas. Resultados: Se incluyeron 2,688 registros durante nueve años consecutivos. El 53.9\% correspondió a hombres, $21.3 \%$ con edades de dos a cinco años y $20.3 \%$ de seis meses a dos años. El $63.5 \%$ de los procedimientos fue electivo. La prevalencia de complicaciones en las primeras 24 horas posteriores al cateterismo fue de $6.7 \%$ (4.2\% menores y $2.4 \%$ mayores). En el $0.8 \%$ de los procedimientos sobrevino la muerte temprana. Los factores relacionados con las complicaciones fueron: edad al momento del cateterismo <28 días (OR, 2.18; IC 95\%, 1.28-3.70), saturación de oxígeno antes del cateterismo <79\% (OR, 2.15; IC 95\%, 1.02-4.53), uso de inotrópicos antes del cateterismo (OR, 3.00; IC 95\%, 1.68-5.33). Las variables incluidas en el modelo explican el $38 \%$ de la varianza de las complicaciones posteriores al cateterismo cardíaco en pacientes menores de 18 años. Conclusiones: En este estudio, el cateterismo cardíaco se vinculó con complicaciones mayores y menores, incluida la muerte. Los factores adjuntos fueron edad menor de 28 días, menor saturación de oxígeno y uso de inotrópicos antes del cateterismo.
\end{abstract}

Palabras clave: Complicaciones. Cateterismo cardíaco. Niño. Mortalidad. Incidencia.

\begin{abstract}
Introduction: Congenital heart diseases are the most common congenital abnormalities in newborns with a prevalence of 1\%. Therapeutic and diagnostic cardiac catheterization has revolutionized the treatment of these diseases; however, it can be associated with complications. Objective: To describe the incidence and type of complications associated with pediatric cardiac catheterization in a reference center for congenital heart defects. Methodology: Retrospective observational study, with analytical intention based on records of cardiac catheterization performed on patients with congenital and acquired heart disease. Results: 2,688 records were included for nine consecutive years. $53.9 \%$ were men, $21.3 \%$ with ages between 2
\end{abstract}

Correspondencia:

*Rafael Lince-Varela

E-mail: rafalince@yahoo.com
Fecha de recepción: 27-06-2020

Fecha de aceptación: 20-11-2020 DOI: $10.24875 /$ ACM.200003191
Disponible en internet: 29-10-2021 Arch Cardiol Mex. 2021;91(4):422-430 www.archivoscardiologia.com 1405-9940 / @ 2020 Instituto Nacional de Cardiología Ignacio Chávez. Publicado por Permanyer. Este es un artículo open access bajo la licencia CC BY-NC-ND (http://creativecommons.org/licenses/by-nc-nd/4.0/). 
and 5 years and $20.3 \%$ between 6 months and 2 years. $63.5 \%$ of the procedures were elective. The prevalence of complications in the first 24 hours after catheterization was 6.7\% (4.2\% minor and $2.4 \%$ major). Early death occurred in $0.8 \%$ of the procedures. Factors associated with complications were age at catheterization <28 days (OR 2.18, 95\% Cl $\left.\left.{ }^{[1} .28-3.70\right]\right)$, precatheter oxygen saturation $\left.<79 \%\left(\mathrm{OR} 2.15,95 \% \mathrm{Cl}^{[1} .02-4.53\right]\right)$, use of pre-catheter inotropics (OR $3.00,95 \% \mathrm{Cl}{ }^{[1} .68$ 5.33]). The variables included in the model explain $38 \%$ of the variance of post-cardiac catheterization complications in patients younger than 18 years. Discussion: Cardiac catheterization is associated with major and minor complications including death. The associated factors were less than 28 days, lower oxygen saturation and use of pre-catheter inotropics.

Key words: Complications. Cardiac catheterization. Child. Mortality. Incidence.

\section{Introducción}

Las cardiopatías congénitas constituyen las anomalías congénitas más comunes en recién nacidos con una prevalencia cercana a 1\%1. Seldinger inició en 1953 la cateterización percutánea en niños ${ }^{2}$ y Lurie la modificó con posterioridad en $1963^{3}$. En las últimas décadas, el cateterismo cardíaco ha revolucionado su tratamiento ${ }^{4}$ gracias a su modalidad no quirúrgica que cumple funciones terapéuticas y diagnósticas al permitir analizar datos fisiológicos y anatómicos ${ }^{5}$. Sin embargo, no es un procedimiento exento de complicaciones, incluida la muerte ${ }^{6}$.

En Latinoamérica es limitada la información acerca de este tema. El objetivo planteado fue determinar la incidencia y el tipo de complicaciones relacionadas con el cateterismo cardíaco en cardiopatías congénitas y adquiridas en un centro de referencia para pacientes con cardiopatías congénitas en la ciudad de Medellín, Colombia.

\section{Material y métodos}

Estudio observacional retrospectivo, con intención analítica a partir de una fuente de información secundaria. Se incluyó a pacientes de ambos sexos, con cardiopatías congénitas y adquiridas tratadas por el grupo de hemodinámica pediátrica y que requirieron cateterismo cardíaco diagnóstico e intervencionista en el período comprendido entre 2009 y 2017. Se excluyó a los pacientes sometidos a cateterismo cardíaco para intervención electrofisiológica. El tamaño de muestra lo determinó la totalidad de los cateterismos practicados de forma consecutiva en la institución en el período de estudio. La variable dependiente fueron las complicaciones vinculadas con el cateterismo en las primeras 24 horas posteriores a éste. Se incluyeron variables sociodemográficas, clínicas y relacionadas con el cateterismo.

Se tomaron medidas para controlar el sesgo de selección (totalidad de registros) y sesgo de información (base construida de forma prospectiva por hemodinamistas y depurada por epidemiólogos; cuando se tuvo dudas se consultó a un cardiólogo pediatra). Además, se realizó control sobre posibles variables de confusión mediante regresión logística.

Se realizó un análisis descriptivo de las variables incluidas. Se buscó un nexo entre las complicaciones posteriores al cateterismo cardíaco y las demás variables incluidas en el estudio. Como medida de asociación se utilizó el OR con su respectivo IC 95\%. Para establecer la relación entre variables cualitativas, se empleó la prueba de la ji cuadrada $\left(\chi^{2}\right)$, de independencia o $p$ de Fisher cuando las frecuencias esperadas eran < 5. Para el análisis de la información se utilizó el software SPSS ${ }^{\circledR}$ versión 21.0 ${ }^{\circledR}$ (SPSS Inc; Chicago. Illinois, USA), licencia amparada. En el modelo de regresión logística binaria se incluyeron variables que en el análisis bivariado mostraron relación estadística y cumplieron el criterio de Hosmer-Lemeshow. El estudio recibió aprobación del Comité de Investigación Institucional y Universitario. Se garantizó la confidencialidad de la información y el uso de ésta con fines académicos. Se tuvieron en cuenta los principios de la Declaración de Helsinki para la investigación en seres humanos.

\section{Resultados}

Se realizaron 2,688 cateterismos entre enero de 2009 y diciembre de 2017, para un promedio de 298 cateterismos por año. El 53.9\% se integró con hombres (Tabla 1).

\section{Diagnóstico}

El diagnóstico principal en el $93.9 \%$ de los casos fueron las cardiopatías congénitas. La más frecuente fue el conducto arterioso persistente en el $28 \%$, seguido por la comunicación interauricular con el $21.99 \%$ y la comunicación interventricular con $19.8 \%$. De las 
Tabla 1. Características sociodemográficas y clínicas de los pacientes sometidos a cateterismo cardíaco pediátrico

\begin{tabular}{|l|c|c|}
\hline & $\mathbf{n = 2 , 6 8 8}$ & $\%$ \\
\hline $\begin{array}{l}\text { Género } \\
\text { - Femenino }\end{array}$ & & \\
\hline - Masculino & 1,238 & 46.1 \\
\hline Edad & 1,450 & 53.9 \\
\hline - $\leq 28$ días & & \\
- 29 días - <6 meses & 235 & 8.7 \\
- 6 meses - <2 años & 367 & 13.7 \\
- 2 años - <5 años & 561 & 20.9 \\
- 5 años - <10 años & 573 & 21.93 \\
- 10 años - <18 años & 442 & 16.4 \\
- Mayor de 18 años & 418 & 15.6 \\
\hline Origen del paciente & 92 & 3.4 \\
\hline - Ambulatorio, electivo & & \\
- Hospitalización & 1,706 & 63.5 \\
- Cuidados intensivos & 622 & 23.1 \\
- Quirófano & 359 & 13.4 \\
\end{tabular}

cardiopatías adquiridas, la principal fue la miocardiopatía dilatada en el 16.1\% (Tabla 2).

\section{Incidencia y tipo de complicaciones posteriores al cateterismo}

Se registraron 179 complicaciones, para una incidencia de $6.7 \%$, el $4.2 \%$ menores y el $2.4 \%$ mayores. Del total de procedimientos, el $0.8 \%$ (22) murió en las primeras 24 horas, pero sólo el $0.11 \%$ se atribuyó directamente al cateterismo.

Las complicaciones que se presentaron en mayor proporción fueron las arritmias en 39.1\% (70/179), la taquicardia supraventricular en $29 \%$, el bloqueo auriculoventricular en $23 \%$, el flúter auricular en $8.5 \%$, la fibrilación ventricular en $8.5 \%$, el bloqueo de rama en $1.4 \%$ y la bradicardia en $29.5 \%$. La segunda complicación más frecuente fue la asistolia con 19.5\% (35/179), diversas con $11.7 \%(21 / 179)$, las relacionadas con el dispositivo con $9.5 \%$ (17/179), el sangrado con $6.3 \%$ (11/179), la embolización del dispositivo con 5.7\% (10/179), las complicaciones vasculares de tipo arterial con 4.0\% (7/179), la hipotensión con 2.5\% (4/179) y el taponamiento cardíaco por manipulación del catéter con $1.7 \%(3 / 179)$.

\section{Procedimiento}

Los cateterismos fueron intervencionistas en $58.3 \%$ y el $6.7 \%$ se realizó luego de que el paciente se sometiera a un procedimiento quirúrgico. Los diagnósticos
Tabla 2. Diagnóstico de cardiopatías congénitas y adquiridas de los pacientes sometidos a cateterismo cardíaco pediátrico

\begin{tabular}{|c|c|c|}
\hline Diagnóstico & $n=2,688$ & $\%$ \\
\hline - Conducto arterioso persistente & 753 & 28 \\
\hline - Comunicación interauricular & 589 & 21.99 \\
\hline - Comunicación interventricular & 533 & 19.8 \\
\hline - Estenosis valvular pulmonar & 340 & 12.6 \\
\hline - Alteraciones de ramas pulmonares & 291 & 10.8 \\
\hline - Coartación de la aorta & 279 & 10.4 \\
\hline $\begin{array}{l}\text { - Síndrome de ventrículo izquierdo } \\
\text { hipoplásico }\end{array}$ & 276 & 10.3 \\
\hline $\begin{array}{l}\text { - Atresia pulmonar sin comunicación } \\
\text { interventricular }\end{array}$ & 260 & 9.7 \\
\hline - Transposición de las grandes arterias & 212 & 7.9 \\
\hline - Defecto septal auriculoventricular & 164 & 6.1 \\
\hline - Atresia tricuspídea & 140 & 5.2 \\
\hline - Estenosis valvular aórtica & 118 & 4.4 \\
\hline $\begin{array}{l}\text { - Atresia pulmonar con comunicación } \\
\text { interventricular }\end{array}$ & 112 & 4.2 \\
\hline - Tetralogía de Fallot & 106 & 3.9 \\
\hline - Doble salida del ventrículo derecho & 106 & 3.9 \\
\hline - Otras & 584 & 21.991 \\
\hline $\begin{array}{l}\text { Anomalías adquiridas } \\
\text { - Miocardiopatía dilatada } \\
\text { - Otras miocardiopatías } \\
\text { - Miocardiopatía no compactada } \\
\text { - Pericarditis constrictiva }\end{array}$ & $\begin{array}{c}434 \\
12 \\
6 \\
1\end{array}$ & $\begin{array}{c}16.1 \\
0.4 \\
0.2 \\
0.01\end{array}$ \\
\hline
\end{tabular}

más frecuentes que exigieron intervencionismo fueron: cierre del conducto arterioso persistente en el $17.2 \%$ de los casos, valvuloplastia pulmonar en $6.7 \%$ y cierre del defecto septal interauricular en el $5.5 \%$ de los casos. La saturación de oxígeno antes del cateterismo fue de $96.3 \%>80 \%$ (Tablas 3 y 4 ).

\section{Análisis de la mortalidad}

De las 22 muertes $(0.81 \%)$ que se presentaron durante las primeras 24 horas luego del cateterismo, el $60 \%$ ocurrió en pacientes menores de 28 días de vida y los procedimientos fueron intervencionistas en $60 \%$. Sólo tres de las muertes tempranas $(0.11 \%)$ se atribuyeron directamente al cateterismo (perforación cardíaca y ruptura de vena y arteria pulmonares). El $41 \%$ de estas muertes se presentó en los pacientes que se programaron para cateterismo en el posoperatorio inmediato. El 31.8\% tenía diagnóstico de síndrome de ventrículo izquierdo hipoplásico (Tabla 5).

\section{Análisis bivariado}

Dentro de las variables incluidas en el estudio se buscaron posibles relaciones con las complicaciones 
Tabla 3. Características hemodinámicas de los cateterismos cardíacos

\begin{tabular}{|c|c|c|}
\hline Cateterismo & $n=2,688$ & $\%$ \\
\hline $\begin{array}{l}\text { Tipo de cateterismo } \\
\text { - Intervencionista } \\
\text { - Diagnóstico }\end{array}$ & $\begin{array}{l}1,568 \\
1,120\end{array}$ & $\begin{array}{l}58.3 \\
41.7\end{array}$ \\
\hline $\begin{array}{l}\text { Cateterismo posoperatorio } \\
\text { - Sí } \\
\text { - No }\end{array}$ & $\begin{array}{c}180 \\
2,508\end{array}$ & $\begin{array}{c}6.7 \\
93.3\end{array}$ \\
\hline $\begin{array}{l}\text { Tipo de procedimiento } \\
\text { - Cierre de conducto arterioso } \\
\text { - Dilatación de válvula pulmonar } \\
\text { - Cierre del defecto septal } \\
\text { interauricular } \\
\text { - Biopsia endomiocárdica } \\
\text { - Stent en arteria pulmonar o vena } \\
\text { sistémica } \\
\text { - Otros procedimientos diversos } \\
\text { - Dilatación de la válvula aórtica } \\
\text { - Auriculoseptostomía con balón } \\
\text { - Angioplastia de arteria pulmonar } \\
\text { - Embolización diversa } \\
\text { - Fontan fenestrado (cierre) } \\
\text { - Angioplastia con balón de } \\
\text { coartación } \\
\text { - Cierre de defecto septal } \\
\text { interventricular } \\
\text { - Dilatación del stent } \\
\text { - Recoartación aórtica con } \\
\text { angioplastia } \\
\text { - Pericardiocentesis }\end{array}$ & $\begin{array}{l}145 \\
130 \\
81 \\
75 \\
72 \\
64 \\
61 \\
51 \\
47 \\
22 \\
17 \\
7 \\
6\end{array}$ & $\begin{array}{c}5.4 \\
4.8 \\
3 \\
2.8 \\
2.7 \\
2.4 \\
2.3 \\
1.9 \\
1.7 \\
0.8 \\
0.6 \\
0.3 \\
0.2\end{array}$ \\
\hline $\begin{array}{l}\text { Tratamiento de la vía respiratoria } \\
\text { - Tubo orotraqueal } \\
\text { - Máscara laríngea } \\
\text { - Ninguno } \\
\text { - Traqueostomía }\end{array}$ & $\begin{array}{c}1,660 \\
1,013 \\
14 \\
1\end{array}$ & $\begin{array}{r}61.8 \\
37.7 \\
0.5 \\
0.01\end{array}$ \\
\hline
\end{tabular}

posteriores al cateterismo. Se encontró un nexo con la edad menor de 28 días, cateterismo posoperatorio, resistencia vascular $>8$ unidades Wood. $\mathrm{m}^{2}$, saturación $<79 \%$, uso de inotrópicos y mayor número de inotrópi$\cos (2-4)$ (Tabla 6).

\section{Análisis multivariado}

Las variables seleccionadas en el análisis bivariado se ingresaron mediante el método Enter a un modelo de regresión logística binaria. Se encontró una relación estadística con edad al momento del cateterismo $<28$ días (OR, 2.18; IC 95\%, 1.28-3.70), saturación de oxígeno antes del cateterismo < 79\% (OR, 2.15; IC 95\%, 1.024.53) y uso de inotrópicos antes del cateterismo (OR, 3.00; IC 95\%, 1.68-5.33). Las variables incluidas en el modelo explican el $38 \%$ de la varianza de las complicaciones posteriores al cateterismo cardíaco (Tabla 7).
Tabla 4. Oxigenación y resistencia vascular pulmonar de los pacientes sometidos a cateterismo cardíaco

\begin{tabular}{|c|c|c|}
\hline Variable & $n=2,688$ & $\%$ \\
\hline $\begin{array}{l}\text { Saturación de } 0_{2} \text { antes del cateterismo } \\
-<64 \% \\
-\geq 80 \% \\
-65-79 \%\end{array}$ & $\begin{array}{c}16 \\
2,588 \\
84\end{array}$ & $\begin{array}{c}0,6 \\
96.3 \\
3.1\end{array}$ \\
\hline $\begin{array}{l}\text { Relación resistencia vascular pulmonar } \\
\text { (IRVP unidades Wood.m) } \\
\text { - Menor a la mitad de la sistémica } \\
\text { - No medida (se asume normal) } \\
\text { - IRVP^ }>1 / 2 \text { la sistémica } \\
\text { - IRVP^ }>\text { la sistémica }\end{array}$ & $\begin{array}{c}1,086 \\
1,547 \\
11 \\
44\end{array}$ & $\begin{array}{c}40.4 \\
57.5 \\
0.5 \\
1.6\end{array}$ \\
\hline 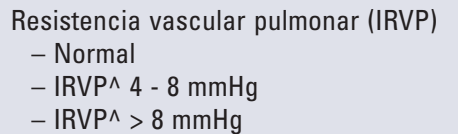 & $\begin{array}{c}2,465 \\
154 \\
69\end{array}$ & $\begin{array}{c}91.7 \\
5.7 \\
2.6\end{array}$ \\
\hline
\end{tabular}

^: índice de resistencia valvular pulmonar.

\section{Discusión}

El principal hallazgo de este estudio fue la determinación de la incidencia de complicaciones tempranas vinculadas con el intervencionismo cardíaco en menores de 18 años en un centro de referencia para cardiopatías congénitas. La incidencia registrada de complicaciones $(6.7 \%)$ se encuentra dentro de los límites informados por otros estudios $(7.3 \%-13 \%)^{7,8}$. En el estudio de Mehta, et al. ${ }^{7}$ la incidencia de complicaciones mayores y menores fue de $7.3 \%$, lo cual es ligeramente superior a los resultados de este estudio, con mayor mortalidad $(0.22 \%$ vs. $0.11 \%$ en este estudio).

\section{Edad}

Los neonatos se han identificado como un grupo de alto riesgo para desarrollar complicaciones secundarias al cateterismo. Otros estudios ${ }^{7,8}$ señalaron que 3 de cada 10 pacientes que morían tempranamente luego del cateterismo eran recién nacidos; en este estudio fueron 6 de cada 10 y éste fue un factor de riesgo independiente para complicaciones tempranas ( $p<0.001$ ). Otro estudio halló la edad menor de un mes como un factor de riesgo adjunto para cualquier complicación (OR, 2.47; IC 95\%, 1.66-3.68), lo cual coincide con este estudio; sin embargo, en este estudio el grupo de edad de mayor riesgo fue el de uno a seis meses de edad (OR, 4.69; IC 95\%, $1.90-11.55)^{9}$. 
Tabla 5. Características clínicas de las muertes relacionadas con el cateterismo cardíaco

\begin{tabular}{|c|c|c|c|c|}
\hline & Edad & Diagnóstico & Procedimiento & Causa de muerte \\
\hline 1 & $1 \mathrm{~d}$ & $\mathrm{SVIH}^{*}$ & $\begin{array}{l}\text { Auriculoseptostomía con balón } \\
\text { estático }\end{array}$ & Perforación, cardiaca, bradicardia \\
\hline 2 & $2 d$ & $\begin{array}{l}\text { Cuerpo extraño intracardíaco (catéter } \\
\text { umbilical) }\end{array}$ & Extracción & Hemorragia pulmonar \\
\hline 3 & $7 d$ & $\mathrm{TGA}^{\wedge}, \mathrm{FOP}^{+}$restrictivo & Auriculoseptostomía con balón & Ruptura de vena pulmonar, asistolia \\
\hline 4 & $8 d$ & Cirugía de Norwood-Sano ERIAP ${ }^{\dagger}$ & $\begin{array}{l}\text { Angioplastia pulmonar } \\
\text { periférica }\end{array}$ & Fibrilación ventricular, asistolia \\
\hline 5 & $11 \mathrm{~d}$ & $\begin{array}{l}\mathrm{TGA}^{\wedge} \text {, cirugía de Jatene, CIV } \text {, oclusión } \\
\text { total ACl× ECMO॥ }\end{array}$ & Diagnóstico & Bradicardia, asistolia \\
\hline 6 & $11 \mathrm{~d}$ & APP $\sin$ CIV & Stent ductal & Bradicardia, asistolia \\
\hline 7 & $13 \mathrm{~d}$ & $\begin{array}{l}\text { EVAo } o^{\ddagger} \text { crítica, CoA } o^{\hat{\imath}} \text { grave, disfunción } \\
\text { ventricular grave }\end{array}$ & Angioplastia aórtica con balón & Bradicardia, asistolia \\
\hline 8 & $15 d$ & AP $\sin$ CIV - PCA & Stent en PCA & $\begin{array}{l}\text { Desplazamiento del stent a arteria } \\
\text { pulmonar, choque hipovolémico }\end{array}$ \\
\hline 9 & $20 \mathrm{~d}$ & SVIH, Norwood-Sano ERIAP & Diagnóstico & Bradicardia, asistolia \\
\hline 10 & $21 \mathrm{~d}$ & TFC, AP ramas no confluentes & Stent ductal & Choque refractario, asistolia \\
\hline 11 & $28 \mathrm{~d}$ & $\begin{array}{l}\text { SVIH, cerclaje de arterias pulmonares, } \\
\text { stent PCA }\end{array}$ & Auriculoseptostomía con bolón & Taquiarritmia \\
\hline 12 & $28 d$ & $\begin{array}{l}\text { SVIH, } P O^{€} \text {, Norwood-Sano, estenosis } \\
\text { crítica de rama izquierda de arteria } \\
\text { pulmonar }\end{array}$ & Diagnóstico & Disfunción ventricular, asistolia \\
\hline 13 & $30 \mathrm{~d}$ & $\mathrm{EM}^{\uparrow}{ }^{\uparrow}, \mathrm{DSVD}^{\downarrow}{ }^{\downarrow}$, FOP restrictivo & Auriculoseptostomía & Bradicardia, asistolia \\
\hline 14 & $2 \mathrm{~m}$ & SVIH - estenosis valvular pulmonar & $\begin{array}{l}\text { Stent en PCA, procedimiento } \\
\text { híbrido }\end{array}$ & Bradicardia, asistolia \\
\hline 15 & $2 \mathrm{~m}$ & $\begin{array}{l}\text { Corazón triauricular izquierdo obstructivo, } \\
\text { HAP!, CIA } \ddagger\end{array}$ & Diagnóstico & Bradicardia, asistolia \\
\hline 16 & $3 \mathrm{~m}$ & $\begin{array}{l}\text { SVIH, Norwood-Sano-Glenn bilateral } \\
\text { bidireccional colateral venovenosa, } \\
\text { hipoxemia grave }\end{array}$ & $\begin{array}{l}\text { Embolización colateral } \\
\text { venovenosa }\end{array}$ & Bradicardia, asistolia \\
\hline 17 & $4 \mathrm{~m}$ & $\begin{array}{l}\mathrm{AT}^{\infty}, \mathrm{PO}, \mathrm{FBTT}^{\beta}, \mathrm{PO} \text { de Glenn bidireccional, } \\
\text { ampliación de CIA, hipoxemia grave }\end{array}$ & Trombectomía en ramas & TEPž, asistolia \\
\hline 18 & $5 \mathrm{~m}$ & $\begin{array}{l}\text { Atresia tricuspídea típica, CIV, CIA, HAP!, } \\
\text { FBTT }\end{array}$ & Diagnóstico & Bradicardia, asistolia \\
\hline 19 & $6 \mathrm{~m}$ & $\begin{array}{l}\text { Aurícula común, canal auriculoventricular, } \\
\text { DSVD }\end{array}$ & Diagnóstico & Disfunción ventricular, asistolia \\
\hline 20 & $10 \mathrm{~m}$ & $\begin{array}{l}\text { POP, AP con CIV, unifocalización con tubo } \\
\text { avalvulado, estenosis RDAP" }\end{array}$ & Diagnóstico & Ruptura de rama pulmonar \\
\hline 21 & $22 \mathrm{~m}$ & $\begin{array}{l}\text { TCGA§, criss-cross - DSVD, CIV } \\
\text { subpulmonar + estenosis pulmonar mixta }\end{array}$ & Diagnóstico & Bradicardia, asistolia \\
\hline 22 & $3 a$ & SVIH, PO, Fontan fenestrado & Diagnóstico & Choque mixto \\
\hline
\end{tabular}

d: días; m: meses; a: años; *: síndrome de ventrículo izquierdo hipoplásico; $\wedge$ : transposición de grandes arterias; +: agujero oval permeable; $†$ : estenosis de la rama izquierda arteria pulmonar; ‡: comunicación interauricular; : comunicación interventricular; $x$ : arteria coronaria izquierda; «: oxigenación de membrana extracorpórea; ¥: estenosis valvular aórtica; î: coartación de la aorta; P: atresia pulmonar; ¿: conducto arterioso permeable; ๔: tetralogía de Fallot; ß: fístula de Blalock-Taussig-Thomas; !: hipertensión arterial pulmonar; ": rama derecha de la arteria pulmonar; §: transposición congénitamente corregida de las grandes arterias; ๆ: doble salida del ventrículo derecho; Ž: tromboembolismo pulmonar; $\uparrow$ : estenosis mitral; $\quad \downarrow$ : disfunción severa del ventrículo derecho; €: postoperatorio; $\infty$ : atresia tricuspídea; 
Tabla 6. Variables sociodemográficas y clínicas relacionadas con complicaciones posteriores al cateterismo cardíaco pediátrico

\begin{tabular}{|c|c|c|c|c|c|}
\hline \multirow[t]{3}{*}{ Variable } & \multicolumn{4}{|c|}{ Complicaciones } & \\
\hline & \multicolumn{2}{|c|}{ Sí } & \multicolumn{2}{|c|}{ No } & \\
\hline & $\mathbf{n}$ & $\%$ & $\mathbf{n}$ & $\%$ & Valor p \\
\hline $\begin{array}{l}\text { Edad } \\
\leq 28 \text { días } \\
>28 \text { días }\end{array}$ & $\begin{array}{c}47 \\
132\end{array}$ & $\begin{array}{l}20 \\
5.4\end{array}$ & $\begin{array}{c}188 \\
2,321\end{array}$ & $\begin{array}{c}80 \\
94.6\end{array}$ & $<0.001^{*}$ \\
\hline $\begin{array}{l}\text { Tipo de cardiopatía } \\
\text { Congénita } \\
\text { Adquirida } \\
\text { Otros }\end{array}$ & $\begin{array}{c}174 \\
5 \\
114\end{array}$ & $\begin{array}{l}6.9 \\
3.0 \\
4.3\end{array}$ & $\begin{array}{c}2,350 \\
159 \\
2,509\end{array}$ & $\begin{array}{l}93.1 \\
97 \\
95.7\end{array}$ & $0.056^{*}$ \\
\hline $\begin{array}{l}\text { Cateterismo PO } \\
\text { Sí } \\
\text { No }\end{array}$ & $\begin{array}{c}30 \\
149\end{array}$ & $\begin{array}{c}16.7 \\
5.9\end{array}$ & $\begin{array}{c}150 \\
2,359\end{array}$ & $\begin{array}{l}83.3 \\
94.1\end{array}$ & $<0.001^{*}$ \\
\hline $\begin{array}{l}\text { Resistencia } \\
\text { vascular } \\
>8 \mathrm{mmHg} \\
\leq 8 \mathrm{mmHg}\end{array}$ & $\begin{array}{c}9 \\
170\end{array}$ & $\begin{array}{c}13,0 \\
6.5\end{array}$ & $\begin{array}{c}60 \\
2,449\end{array}$ & $\begin{array}{c}87 \\
93.5\end{array}$ & $0.031^{\wedge}$ \\
\hline $\begin{array}{l}\text { Relación resistencia } \\
\text { >Sistémica } \\
\text { Otros }\end{array}$ & $\begin{array}{c}5 \\
174\end{array}$ & $\begin{array}{l}11.4 \\
6.6\end{array}$ & $\begin{array}{c}39 \\
2,470\end{array}$ & $\begin{array}{l}88.6 \\
93.4\end{array}$ & $0.207 \wedge$ \\
\hline $\begin{array}{l}\mathrm{SaO}_{2} \\
\leq 79 \% \\
>80 \%\end{array}$ & $\begin{array}{c}13 \\
166\end{array}$ & $\begin{array}{l}13 \\
6.4\end{array}$ & $\begin{array}{c}87 \\
2,422\end{array}$ & $\begin{array}{c}87 \\
93.6\end{array}$ & $0.010^{*}$ \\
\hline $\begin{array}{l}\text { Uso de inotrópicos } \\
\text { Sí } \\
\text { No }\end{array}$ & $\begin{array}{l}88 \\
91\end{array}$ & $\begin{array}{c}22.6 \\
4\end{array}$ & $\begin{array}{c}301 \\
2,208\end{array}$ & $\begin{array}{c}77.4 \\
96\end{array}$ & $<0.001^{*}$ \\
\hline 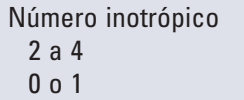 & $\begin{array}{c}29 \\
150\end{array}$ & $\begin{array}{c}29.3 \\
5.8\end{array}$ & $\begin{array}{c}70 \\
2,439\end{array}$ & $\begin{array}{l}70.7 \\
94.2\end{array}$ & $<0.001^{*}$ \\
\hline
\end{tabular}

*: ji cuadrada de Pearson; ^: prueba exacta de Fisher; $\mathrm{SaO}_{2}$ : saturación de oxígeno; PO: posoperatorio.

\section{Tipo de complicaciones}

En el estudio de Mehta, et al.,(7) los efectos adversos fueron en orden de frecuencia las complicaciones vasculares $(32.4 \%)$, seguidas de las arritmias $(23 \%)$, anomalías diversas (17.3\%) y el sangrado (8.6\%). En este estudio, las tres complicaciones más frecuentes fueron las arritmias, los episodios vasculares y el paro cardíaco (asistolia). Se identificó una menor frecuencia en este estudio de sangrado ( $6.3 \%$ vs. $8.6 \%$ ).

\section{Arritmias}

En un estudio turco ${ }^{10}$, el cual incluyó 2,693 cateterismos cardíacos diagnósticos en niños, el $7 \%$ presentó arritmias, el $62 \%$ de ellas correspondió a bradiarritmias
Tabla 7. Modelo multivariado: variables ajustadas que mostraron relación con las complicaciones tempranas del cateterismo cardíaco

\begin{tabular}{|l|c|c|c|c|}
\hline & \multirow{2}{*}{ Valor p } & OR & \multicolumn{2}{|c|}{ IC 95\% } \\
\cline { 4 - 5 } & & & $\begin{array}{c}\text { Límite } \\
\text { inferior }\end{array}$ & $\begin{array}{c}\text { Límite } \\
\text { superior }\end{array}$ \\
\hline Edad $<28$ días & $<0.000$ & 2.45 & 1.63 & 3.67 \\
\hline $\begin{array}{l}\text { Cateterismo } \\
\text { posoperatorio }\end{array}$ & 0.414 & 0.741 & 0.361 & 1.521 \\
\hline $\begin{array}{l}\text { Resistencia } \\
\text { vascular }>8 \\
\text { unidades Wood.m }\end{array}$ & 0.161 & 2.101 & 0.744 & 5.929 \\
\hline $\begin{array}{l}\text { Sa0 } \\
\text { Sa }<79 \%\end{array}$ & 0.044 & 2.108 & 1.115 & 3.986 \\
\hline $\begin{array}{l}\text { Uso de inotrópico } \\
\text { antes del } \\
\text { cateterismo }\end{array}$ & $<0.000$ & 5.258 & 3.694 & 7.485 \\
\hline
\end{tabular}

y $38 \%$ a taquiarritmias. Dentro de las bradiarritmias, la más común fue la anormalidad en la conducción $\mathrm{AV}$ en el $47 \%$ del total de las arritmias y la taquiarritmia más común fue la supraventricular, observada en el $28 \%$ del total de arritmias. Mehta, et al., ${ }^{7}$ publicaron una incidencia de $30 \%$ para la taquicardia supraventricular y $28 \%$ para el bloqueo $\mathrm{AV}$, lo cual es similar a los resultados de los autores, y las arritmias fueron la principal complicación, con 4 de cada 10 complicaciones y predominio ligero de la taquicardia supraventricular sobre la bradicardia. La recuperación espontánea ocurrió en el $82 \%$ en tanto que en el resto de los pacientes fue necesaria la implantación de un marcapasos transitorio. La mayoría de los pacientes con taquicardia ventricular mejoró con desfibrilación y uno de ellos requirió apoyo con membrana extracorpórea. En este estudio, la mayor parte de las arritmias se resolvió de modo espontáneo ${ }^{7}$.

La cardiopatía que se vinculó con más arritmias fueron la anomalía de Ebstein (37\% del total de los pacientes con esta cardiopatía), la transposición corregida de grandes arterias (24\% del total de transposiciones) y la doble salida del ventrículo izquierdo ( $19 \%$ del total de esta cardiopatía). En este caso, las cardiopatías que más se vincularon con arritmias fueron: síndrome de ventrículo izquierdo hipoplásico (11.95\%) y atresia pulmonar sin CIV $(8.88 \%)^{7}$.

\section{Complicaciones vasculares}

En un estudio prospectivo, Vitiello, et al., ${ }^{11}$ tomaron 4,952 procedimientos consecutivos y encontraron que 
las principales complicaciones vinculadas con el cateterismo cardíaco diagnóstico, intervencionista o electrofisiológico fueron las de tipo vascular con 3.8\% en el total de los procedimientos y $7.3 \%$ en los menores de un año de edad. La trombosis arterial fue la complicación más frecuente, detectada por disminución o ausencia de pulso en 165 pacientes. Sin embargo, como la verdadera incidencia de complicaciones arteriales no puede conocerse sólo con la exploración física, en un protocolo de Kocis, et al., ${ }^{12}$ se identificó una incidencia de compromiso del flujo en el $32 \%$ de los pacientes y de éstos sólo el $57 \%$ se había detectado por exploración clínica

\section{Asistolia}

Odegard, et al., ${ }^{13}$ realizaron un estudio entre 2004 y 2009, el cual incluyó todos los paros cardíacos ocurridos durante el cateterismo cardíaco por cardiopatías congénitas en 7,289 procedimientos de cateterismo. Se registraron 70 paros cardíacos para una incidencia de $0.96 \%$ sobre el total de los cateterismos; de estos episodios, el $69 \%$ se reanimó de forma exitosa, en tanto que el $26 \%$ necesitó oxigenación por membrana extracorpórea y en $6 \%$ sobrevino la muerte. El comienzo de la arritmia precedió al inicio de la asistolia en el $54 \%$ de los casos y se encontró un nexo con intervencionismo y menor edad $(p<0.001)$ para ambos factores. En este estudio, la asistolia se presentó en una de cada cinco complicaciones, en dos tercios finalizó con la muerte y en un tercio se reanimaron de manera exitosa.

\section{Relación con la manipulación del catéter}

La manipulación del catéter por sí misma puede precipitar complicaciones como la perforación cardíaca, informada con una incidencia de $5.5 \%$; en el estudio de los autores se presentó una $(n=1)$ perforación cardíaca que llevó a la muerte; la incidencia de embolización fue de $3 \%$, menor a lo ocurrido en este estudio ${ }^{7}$.

\section{Sangrado}

En el estudio de Vitiello, et al., ${ }^{11}$ se comunicaron cinco casos graves de sangrado en 1,081 procedimientos y 25 casos de sangrado menor de tipo hematoma. Esto representó una incidencia total de $2.77 \%$ sobre el total de los procedimientos y de $6.8 \%$ sobre el total de las complicaciones, lo cual es similar a lo encontrado en este estudio, pero a diferencia de ellos todos los episodios fueron menores.

\section{Uso de inotrópicos}

Un estudio mexicano (2016) encontró que los inotrópicos (OR, 7.0), edad menor de un año (OR, 5.45) y el ingreso urgente $(O R, 1.2)$ eran factores relacionados con las complicaciones posteriores al cateterismo ${ }^{8}$, lo cual coincide con este estudio que reconoció el uso de inotrópicos antes del cateterismo como un factor de riesgo independiente para complicaciones.

\section{Saturación de oxígeno antes del cateterismo}

La hipoperfusión tisular como resultado de un déficit circulatorio se ha identificado como un poderoso factor de riesgo para el desarrollo de insuficiencia de órgano y muerte ${ }^{14}$. En un estudio retrospectivo multicéntrico que incluyó 8,111 procedimientos ${ }^{10}$ se encontró que la baja saturación de oxígeno en prematuros y neonatos era un factor de riesgo para complicaciones tempranas. En el estudio de los autores, este fue un factor de riesgo independiente acompañado de complicaciones en todos los grupos de edad.

\section{Experiencia del centro}

Un estudio que comparó las complicaciones tempranas vinculadas con el cateterismo cardíaco en niños, el cual incluyó a 44 centros con 42,219 sujetos y 68,463 procedimientos, identificó que el incremento del volumen de cateterismo de los centros reducía el riesgo de complicaciones. El riesgo de muerte relacionado con cateterismo en un centro con un volumen promedio de 100 cateterismos/año, con riesgo ajustado de complicaciones, tuvo OR de $0.14 \%$ e IC $95 \%$ de $0.08-0,23 \%$, un centro con un promedio de 300 cateterismos registró un OR de $0.11 \%$ e IC $95 \%$ de $0.07-0.16 \%$ ) y los centros con 500 cateterismos/año un OR de $0.08 \%$ e IC $95 \%$ de $0.05-0.13 \%{ }^{15}$. La mediana de volumen de cateterización en este estudio fue de 264 procedimientos/año y la mediana de 297 (DE, 171); esta media es similar a la de los autores de 298.6 cateterismos/año (DE, 40) con siete complicaciones por cada 100 cateterismos, lo cual se encuentra en los límites señalados.

\section{Mortalidad relacionada con cardiopatías congénitas}

Antes de la cirugía cardíaca, menos de 50 años atrás, sólo el $30 \%$ de los niños con enfermedad cardíaca congénita grave sobrevivía a la adultez. En la 
actualidad, la mortalidad vinculada con la cirugía cardíaca en un estudio que incluyó a 1,550 pacientes fue de $3.2 \%{ }^{16}$.

De modo similar, 35 años antes, la mortalidad secundaria a cateterismo cardíaco en niños era de $3.8 \%$ a las 24 horas, $8.3 \%$ a las 48 horas y $13.5 \%$ a la sema$\mathrm{na}^{17}$. Los informes actuales de mortalidad varían ampliamente: Zahn, et al., $0 \%{ }^{18}$; Bennet, et al., $0.08 \%{ }^{19}$; Vitiello, et al., $0.14 \%$; ;avli, et al., $0.4 \%^{20}$, y Safaa, et al., $0.7 \%{ }^{21}$, lo que sitúa a este centro en el límite superior. Algunos factores que puede contribuir a esto son la falta de disponibilidad de ECMO para el apoyo de los pacientes críticos y la tecnología implementada en fecha reciente en la institución y en este grupo de pacientes fallecidos (sólo uno de los pacientes la recibió). Otros aspectos más difíciles de determinar son el retraso administrativo o geográfico que tienen los pacientes para acceder a los recursos asistenciales, lo cual puede contribuir al peor estado hemodinámico al momento del procedimiento, además del tipo de pacientes que interviene cada centro asistencial puesto que a mayor experiencia del grupo que interviene a niños con cardiopatías congénitas mayor es la complejidad de los pacientes atendidos); en este aspecto, el centro de los autores tiene un programa de ventrículo único, lo cual explica que uno de cada tres pacientes fallecidos tenga diagnóstico de ventrículo izquierdo hipoplásico.

\section{Limitaciones}

Como limitaciones debe mencionarse el carácter retrospectivo del estudio y que éste no se diseñó para determinar factores vinculados con las complicaciones del cateterismo, por lo que puede subestimarse la incidencia de complicaciones raras.

\section{Conclusiones}

El cateterismo cardíaco pediátrico es un procedimiento invasivo no exento de complicaciones, incluida la muerte. Los neonatos, la inestabilidad hemodinámica, la baja saturación de oxígeno y los procedimientos intervencionistas fueron factores determinantes en la incidencia de complicaciones y mortalidad temprana.

Este estudio pionero en el medio permite establecer una línea de base para futuras comparaciones a partir de nuevos estudios, además de identificar algunos factores susceptibles de modificación para lograr una disminución de las complicaciones adjuntas.

\section{Financiamiento}

Esta investigación no ha recibido ninguna beca específica de agencias de los sectores público, comercial o sin ánimo de lucro.

\section{Conflicto de intereses}

$$
\text { Ninguno. }
$$

\section{Responsabilidades éticas}

Protección de personas y animales. Los autores declaran que para esta investigación no se han realizado experimentos en seres humanos ni en animales.

Confidencialidad de los datos. Los autores declaran que han seguido los protocolos de su centro de trabajo sobre la publicación de datos de pacientes.

\section{Derecho a la privacidad y consentimiento infor-} mado. Los autores han obtenido el consentimiento informado de los pacientes o sujetos referidos en el artículo. Este documento obra en poder del autor de correspondencia.

\section{Bibliografía}

1. Tennant PW, Pearce MS, Bythell M, Rankin J. 20-year survival of children born with congenital anomalies: a population-based study. The Lancet. 20 de febrero de 2010;375(9715):649-56.

2. Seldinger SI. Catheter replacement of the needle in percutaneous arteriography; a new technique. Acta Radiol mayo de 1953;39(5):368-76.

3. Lurie PR, Armer RM, Klatte EC. PERCUTANEOUS GUIDE WIRE CATHETERIZATION--DIAGNOSIS AND THERAPY. Am J Dis Child 1960. agosto de 1963;106:189-96.

4. Feltes TF, Bacha E, Beekman RH, Cheatham JP, Feinstein JA, Gomes AS, et al. Indications for Cardiac Catheterization and Intervention in Pediatric Cardiac Disease: A Scientific Statement From the American Heart Association. Circulation. 7 de junio de 2011;123(22):2607-52.

5. Qureshi SA, Redington AN, Wren C, Ostman-Smith I, Patel R, Gibbs JL, et al. Recommendations of the British Paediatric Cardiac Association for therapeutic cardiac catheterisation in congenital cardiac disease. Cardiol Young. noviembre de 2000;10(6):649-67.

6. Cassidy SC, Schmidt KG, Van Hare GF, Stanger P, Teitel DF. Complications of pediatric cardiac catheterization: a 3-year study. J Am Coll Cardiol. mayo de 1992;19(6):1285-93.

7. Mehta R, Lee K-J, Chaturvedi R, Benson L. Complications of pediatric cardiac catheterization: a review in the current era. Catheter Cardiovasc Interv Off J Soc Card Angiogr Interv. 1 de agosto de 2008;72(2):278-85.

8. Zepeda-Arámbula A, Gutiérrez-Cobian L, Villatoro-Fernández JL, Pacheco-López SL, Tlacuilo-Parra A. Adverse events during diagnostic and therapeutic pediatric cardiac catheterization. Rev Médica Inst Mex Seguro Soc. 15 de marzo de 2017;54(S3):276-83.

9. O'Byrne ML, Kennedy KF, Kanter JP, Berger JT, Glatz AC. Risk Factors for Major Early Adverse Events Related to Cardiac Catheterization in Children and Young Adults With Pulmonary Hypertension: An Analysis of Data From the IMPACT (Improving Adult and Congenital Treatment) Registry. J Am Heart Assoc. 28 de febrero de 2018;7(5):e008142.

10. Kasar T, Tanıdır IC, Öztürk E, Gökalp S, Tunca Şahin G, Topkarcı MA et al. Arrhythmia during diagnostic cardiac catheterization in pediatric patients with congenital heart disease. Turk Kardiyol Dernegi Arsivi. 1 de diciembre de 2018;46(8):675-82.

11. Vitiello R, W McCrindle B, Nykanen D, M Freedom R, Benson L. Complications Associated Wtih Pediatric Cardiac Catheterization. J Am Coll Cardiol. 1 de diciembre de 1998;32:1433-40.

12. Kocis KC, Snider AR, Vermilion RP, Beekman RH. Two-dimensional and Doppler ultrasound evaluation of femoral arteries in infants after cardiac catheterization. Am J Cardiol. 15 de marzo de 1995;75(8):642-5. 
Arch Cardiol Mex. 2021;91(4)

13. Odegard KC, Bergersen L, Thiagarajan R, Clark L, Shukla A, Wypij D, et al. The frequency of cardiac arrests in patients with congenital hear disease undergoing cardiac catheterization. Anesth Analg. enero de 2014:118(1):175-82.

14. Hu BY, Laine GA, Wang S, Solis RT. Combined central venous oxygen saturation and lactate as markers of occult hypoperfusion and outcome following cardiac surgery. J Cardiothorac Vasc Anesth. febrero de 2012;26(1):52-7.

15. O'Byrne ML, Glatz AC, Shinohara RT, Jayaram N, Gillespie MJ, Dori Y et al. Effect of center catheterization volume on risk of catastrophic adverse event after cardiac catheterization in children. Am Heart J. junio de 2015;169(6):823-832.e5

16. Berger JT, Holubkov R, Reeder R, Wessel DL, Meert K, Berg RA, et al. Morbidity and mortality prediction in pediatric heart surgery: Physiological profiles and surgical complexity. J Thorac Cardiovasc Surg. agosto de 2017;154(2):620-628.e6.
17. Cohn HE, Freed MD, Hellenbrand WF, Fyler DC. Complications and mortality associated with cardiac catheterization in infants under one year: a prospective study. Pediatr Cardiol. 1985;6(3):123-31.

18. Zahn EM, Dobrolet NC, Nykanen DG, Ojito J, Hannan RL, Burke RP. Interventional catheterization performed in the early postoperative period after congenital heart surgery in children. J Am Coll Cardiol. 7 de abril de 2004;43(7):1264-9.

19. Bennett D, Marcus R, Stokes M. Incidents and complications during pediatric cardiac catheterization. Paediatr Anaesth. diciembre de 2005; 15(12):1083-8.

20. Tavli V, Kayhan B, Okur FF, Kirman M, Tekdoğan M. Complications of pediatric cardiac catheterization: 18-month study. Turk J Pediatr. diciembre de 2000;42(4):294-7.

21. Ali SH. Adverse events in pediatric cardiac catheterization: Initial experience of Sohag university hospital. Egypt Heart J. 2016;1(68):45-51 